\title{
Solid State Sintered 3-D Printing Component by Using Inkjet (Binder) Method
}

\author{
Robert FRYKHOLM ${ }^{1}$, Yoshinobu TAKEDA ${ }^{2}$, Bo-Göran ANDERSSON ${ }^{1}$ and Ralf CARLSTRÖM ${ }^{1}$ \\ ${ }^{1}$ Höganäs AB, SE 263-83 Höganäs, Sweden. \\ ${ }^{2}$ Höganäs Japan K.K., Akasaka Shasta East Building, 4-2-19, Akasaka, Minato-ku, Tokyo 107-0052, Japan.
}

Received November 27, 2015; Revised January 12, 2016; Accepted January 14, 2016

\begin{abstract}
Additive manufacturing of metal components is commonly performed by laser or electron beam melting for direct consolidation of powders, but there are also other techniques available, e.g. the ink-jet on powder bed used by Digital Metal ${ }^{\circledR}$. Höganäs Digital Metal has been involved in additive manufacturing since 2010, with a technology that utilizes separate forming and consolidation processes. Precision inkjet on powder bed is used for production of green components, and these components are then sintered to obtain final density and strength. The technique is successfully used to produce components in 316L stainless steel, but basically all types of metals or alloys, available as powders with suitable morphology and particle size can be used to print components. The following sintering process then can be optimized for each individual alloy, making processing of a wide selection of alloys possible. In this study, possibilities and challenges with different alloys are discussed.
\end{abstract}

\section{KEY WORDS}

stainless steels, hot isostatic pressing, densification, titanium alloys

\section{Introduction}

Additive manufacturing is a rapidly growing business. Most of the revenue derived from the technology is attributed to production of plastic parts, but also the production of metal parts by $3 \mathrm{D}$ printing is steadily increasing. Application range covers areas all the way from non-critical jewelry to high performance medical and aerospace parts. The demands on performance, surface quality, and detail level are steadily increasing. The principle is that components are built directly, layer by layer, from 3D CAD data. For metallic components, laser or electron beam is used for direct consolidation of powders, but there are also other techniques available, e.g. the inkjet on powder bed utilized by Digital Metal.

In the Digital Metal process components are built up layer by layer derived from an original $3 \mathrm{D}$ or $\mathrm{CAD}$ file. The file is prepared and sliced into 2D files corresponding to $42 \mu \mathrm{m}$ thick layers of the component, $42 \mu \mathrm{m}$ being the resolution limit for the technique at present. These files are used as input to the printer. In the building stage, a layer of $42 \mu \mathrm{m}$ powder is applied in the build box, and the printer passes over the surface and deposits "ink" on relevant spots based on information from the 2D file. When printing of the layer is finished, the bottom of the build box is lowered, a new powder layer is applied, and the printing process is repeated layer by layer until the component is formed in accordance with the

\footnotetext{
* Corresponding author, E-mail:robert.frykholm@hoganas.com
}

original 3D file. The process is performed at room temperature, and no consideration of thermal conductivity needs to be taken. This means that the use of the build box volume can be maximized, as the components can be packed tightly. Furthermore, there is in principal no need for building support structures during printing since the components are sufficiently supported by the powder bed in the build box. No distortions due to heat gradients will occur. Building time is approximately one hour per height centimeter at present, and the surface printed has little effect on the building time, meaning it basically takes just as long to print a full packed box as it takes to print a few small components. The principle of the technique is presented in Fig. 1. As printing of the metal powders occurs at room temperature followed by a separate

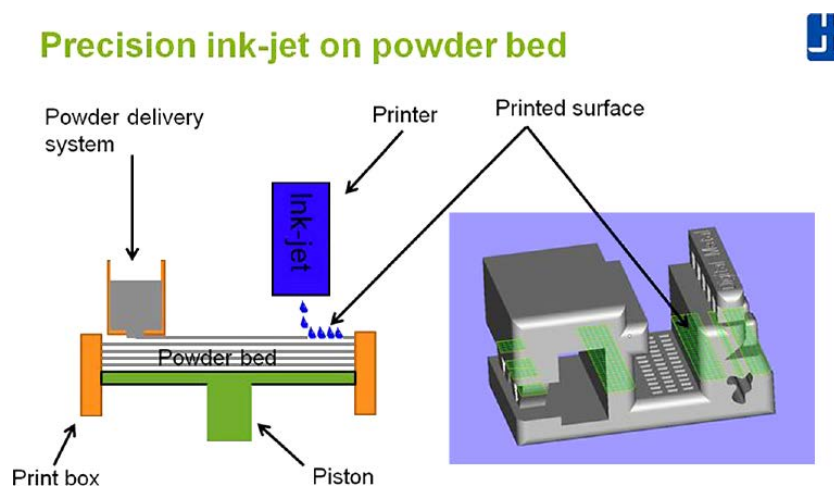

Fig. 1 Overview of the Digital Metal printing process. 
sintering, there is no heat involved during building and printing can be performed without protective gas. Since no melting takes place during building, green components can be produced with very high detail levels and tolerances. Also, since building and heat treatment are separate processes, this allows for a wide selection of materials, where each process step can be optimized for each material. Virtually all materials that can be sintered, can also be utilized in the Digital Metal process, as long as the material is available as powder with adequate properties.

Powder properties that can affect the final properties of the components are e.g. particle size, morphology, density, tap density, and flowability. In order to reach a high component resolution, the powder particles have to be sufficiently small, to match the building resolution of $42 \mu \mathrm{m}$. There is need for sufficient tap density of the powder in order to get good packing of the powder layers and high green density in the components. If tap density is not high enough, the green components might have high porosity levels. This leads to high shrinkage during sintering, difficulties to reach high final density, and difficulties to keep tolerances. Spherical particle morphology is to prefer, but not completely necessary. Somewhat uneven particles might improve green strength, but might also be a disadvantage regarding uniform density. Selection of proper powder is important for maximum utilization of the possibilities with the Digital Metal process, and when the process is performed correctly the technology can offer tolerances down to $50 \mu \mathrm{m}$ or better, and hole and wall thicknesses down to $200 \mu \mathrm{m}$ at the present time.

To be able to handle the green components after building, it is important that the organic component provides sufficient green strength. For this, surface properties of the powder particles can be important. The interaction between organic component and powder, such as wetting behavior, might affect the building process, and the organic component might have to be adjusted for the material selected. Some materials, e.g. $\mathrm{Cu}$, might easily oxidize at relatively low temperatures, and then caution has to be taken regarding temperature and water content. A risk otherwise is that oxidation might glue particles together, making removal of excess powder more difficult.

Once the green components are cleaned from excess loose powder they can be sintered. For each material, the heat treatment process has to be optimized for binder removal and densification. The final result is quite similar to what is obtained from metal injection molding. If printing and sintering is performed correctly, a component with high density will be possible to obtain, and the material will have isotropic properties. Surface roughness after sintering is normally $\mathrm{R}_{\mathrm{a}} 6$ but can easily be improved with traditional surface treatment processes to $\mathrm{R}_{\mathrm{a}} 3$ or better.

\section{Materials}

As mentioned, the Digital Metal technique offers the possibility to use a wide selection of materials, where different materials are more or less difficult to process, possessing different challenges. While most of the production today is based on stainless $316 \mathrm{~L}$ steel, successful trials have also been performed with e.g. 17-4PH steel, titanium, and copper. The printing process is the same for all materials, but binder choice might vary in order to optimize the building.

$2.1316 \mathrm{~L}$

$316 \mathrm{~L}$ can be processed to close to full density with correct sintering settings. The material is then sintered at high temperature, close to solidus temperature. When sintered in vacuum, a partial pressure of Ar is applied. This is done to reduce loss of $\mathrm{Cr}$ from the surface, which otherwise might occur due to the vapor pressure of $\mathrm{Cr}$. Loss of $\mathrm{Cr}$ has to be prevented in order to maintain the corrosion resistance of the steel. Typical porosity and microstructure can be found in Fig. $2^{1)}$. This is what can be expected after standard processing, with sintering at $1360-1380{ }^{\circ} \mathrm{C}$. Since sintering is performed at high temperature, a large amount of delta-ferrite is present. This is beneficial from diffusion point of view. After cooling there might be some residues of ferrite in the material. This is well known from MIM, and investigations have shown that the effect on mechanical performance and corrosion resistance is rather small up to $8 \%$ of delta ferrite ${ }^{2)}$. To reach higher densities the sintering temperature could be increased, but this is normally not done since the higher temperature will have a negative effect on tolerances. Typically, for 316L steel, the aim is set to $96-97 \%$ density, which can be obtained with good dimensional stability maintained.

\section{$2.2 \quad 17-4 \mathrm{ph}$}

$17-4 \mathrm{ph}$ does not require any different treatment compared to 3161 regarding printing and sintering. It is a material that easily can be incorporated in the Digital Metal process. Since it is a material intended for heat treatment, secondary operations could be required for final properties. In Fig. 3 typical pore structure after sintering is shown. 2.3 Titanium alloys

Titanium is a material that combines low weight with high mechanical performance. It is also a material of great interest for biomedical applications. The most common Ti alloy used is

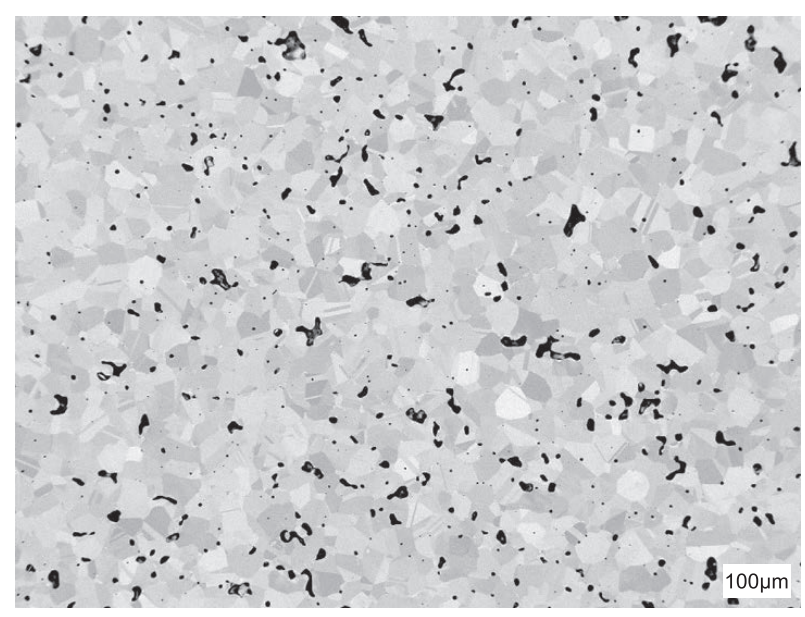

Fig. 2 Porosity and microstructure after standard sintering of $316 \mathrm{~L}\left(\right.$ after $\left.^{1)}\right)$. 


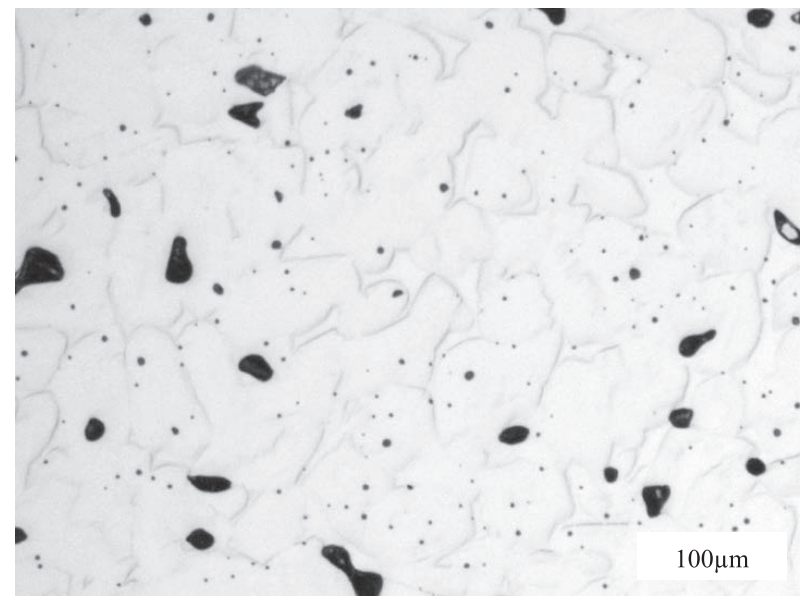

Fig. 3 Porosity and microstructure after standard sintering of 17-4PH.

the Ti-6Al-4V, and it is therefore a material tested in this Digital Metal process.

Performance of titanium is very sensitive to impurity levels. At sintering temperature Ti dissolves $\mathrm{O}, \mathrm{C}$, and $\mathrm{N}$, and once these elements are present in the Ti matrix, they will be impossible to remove. Already at low levels these elements will have influence on the mechanical performance of the material. Up to a certain level they can enhance strength, but with increasing levels ductility drastically decreases, and the material becomes brittle. This makes Ti somewhat more complicated to handle in the process, since the binder contains $\mathrm{C}$, and almost all $\mathrm{C}$ from the binder is necessary to remove already at low temperature to avoid diffusion into the Ti matrix, and deterioration of mechanical properties as a consequence. Work has been performed in order to minimize $\mathrm{C}$-content at low temperature, to prevent $\mathrm{C}$-residues from forming $\mathrm{TiC}$ at high temperature. Processing has been performed at different temperatures, and in different atmospheres, and it has been found possible to keep $\mathrm{C}$ at very low levels.

Sintering of titanium is preferably performed in vacuum. Fig. 4 shows porosity and microstructure after sintering at three different temperatures, 1250,1300 , and $1350{ }^{\circ} \mathrm{C}$ for $2 \mathrm{~h}$. Porosity levels after these sintering trials were between $7-10 \%$. Sintering parameters can be adjusted to deliver higher densities, but if too high sintering temperature is applied, there is a risk that grain growth will have a negative effect on the mechanical properties. As can be seen in the
Table 1 Impurity levels after sintering, and limits according to standards.

\begin{tabular}{lccccc}
\hline & $1250{ }^{\circ} \mathrm{C}$ & $1300{ }^{\circ} \mathrm{C}$ & $1350{ }^{\circ} \mathrm{C}$ & $\begin{array}{c}\text { ISO 22068 } \\
\text { MIM }\end{array}$ & Grade 5 \\
\hline $\mathrm{C}$ (wt.\%) & 0.08 & 0.06 & 0.03 & $<0.2$ & 0.08 \\
$\mathrm{O}$ (wt.\%) & 0.20 & 0.22 & 0.26 & $<0.4$ & 0.2 \\
\hline
\end{tabular}

micrographs, with higher sintering temperature, the microstructure becomes somewhat coarser, and at too high temperatures this coarsening can have a negative effect on properties.

Table 1 shows $\mathrm{C}$ and $\mathrm{O}$ levels after sintering. The table also shows maximum levels according to MIM standard for Ti-6Al$4 \mathrm{~V}$, and for Ti grade 5 . The MIM standard has quite wide impurity range, and can easily be fulfilled. The Grade $5 \mathrm{C}$-limit is possible to fulfill at all temperatures, but oxygen levels are somewhat too high after sintering at 1300 and $1350{ }^{\circ} \mathrm{C}$. It is, however, important to remember that this oxygen level is a result of the sintering process. It is not oxygen that has been introduced in the furnace with the component, and with better vacuum it should be possible to reduce O-level further, and produce Ti components with high purity levels with the Digital Metal process. Also, the trials in this study were performed with only a small load of specimens in the furnace, which is not optimal for Ti sintering since virtually all $\mathrm{O}$ available will diffuse into the components. With higher furnace load there will be less oxygen available per component.

\subsection{Copper and silver}

Copper and silver are two materials which behave quite similar in heat treatments. When sintered they are very sensitive to heating rate. It is important that surface oxides are reduced before pore closure, otherwise residues might induce pore swelling ${ }^{3)}$. Since applications for $\mathrm{Cu}$ and $\mathrm{Ag}$ often involve thermal or electrical conductivity, density is of high importance, and porosity must be minimized. According to MPIF standard 35, minimum density for MIM Cu is $8.5 \mathrm{~g} / \mathrm{cm}^{3}$, and typical value is $8.75 \mathrm{~g} / \mathrm{cm}^{3}$. This corresponds to approximately 95 and $98 \%$ of theoretical density, respectively. Both $\mathrm{Cu}$ and $\mathrm{Ag}$ also easily oxidize at low temperature, which makes them sensitive to handling before sintering. Heat has to be avoided in the printing process. Otherwise powder particles might bond by oxidation, and removal of excessive powder will be impossible. Processes for both $\mathrm{Cu}$ and $\mathrm{Ag}$ are under development at Höganäs Digital Metal. By proper choice of ink and processing
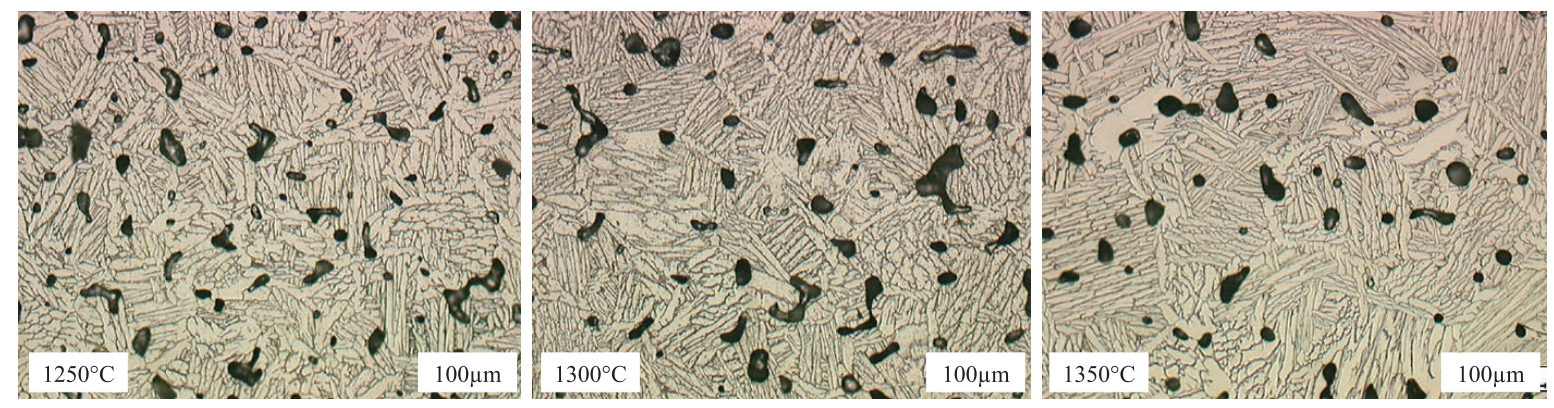

Fig. 4 Overview of the microstructure of the Ti-6Al-4V specimens after sintering for $2 \mathrm{~h}$ in vacuum. 


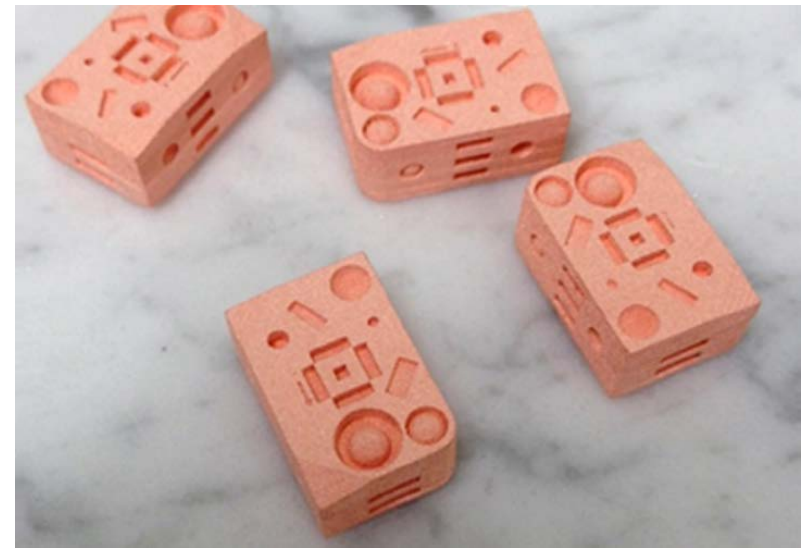

Fig. $5 \mathrm{Cu}$-components produced by inkjet on powder bed technique.

parameters, both $\mathrm{Cu}$ and $\mathrm{Ag}$ components have been printed. The system utilized allows for effective removal of excess powder, and offers possibility to obtain high quality green components. Next step in the development is to optimize the heat treatment to ensure effective debinding and high densification. Fig. 5 shows an example of printed and sintered $\mathrm{Cu}$ components.

\section{Density optimization}

With standard Digital Metal processing, a density level of approximately $96 \%$ of theoretical is achieved for 316L. Similar results are obtained for $17-4 \mathrm{PH}$, while density at present is somewhat lower for $\mathrm{Ti}, \sim 93 \%$. If higher densities are required, HIP (Hot Isostatic Pressing) can be performed. A prerequisite then is that the density of the sintered specimen is high enough not to have a structure with open porosity. This is very important. If the porosity is open and connected, HIP will have no effect since pressure will be applied uniformly through the specimen on all pore surfaces. With closed porosity, pressure will be applied uniformly only on the outer component surfaces, and thereby induce densification. With this technique almost full density can be reached. Examples for 316L, 17-4PH and Ti-6Al-4V are presented in Fig. 6. All materials respond well to the treatment,
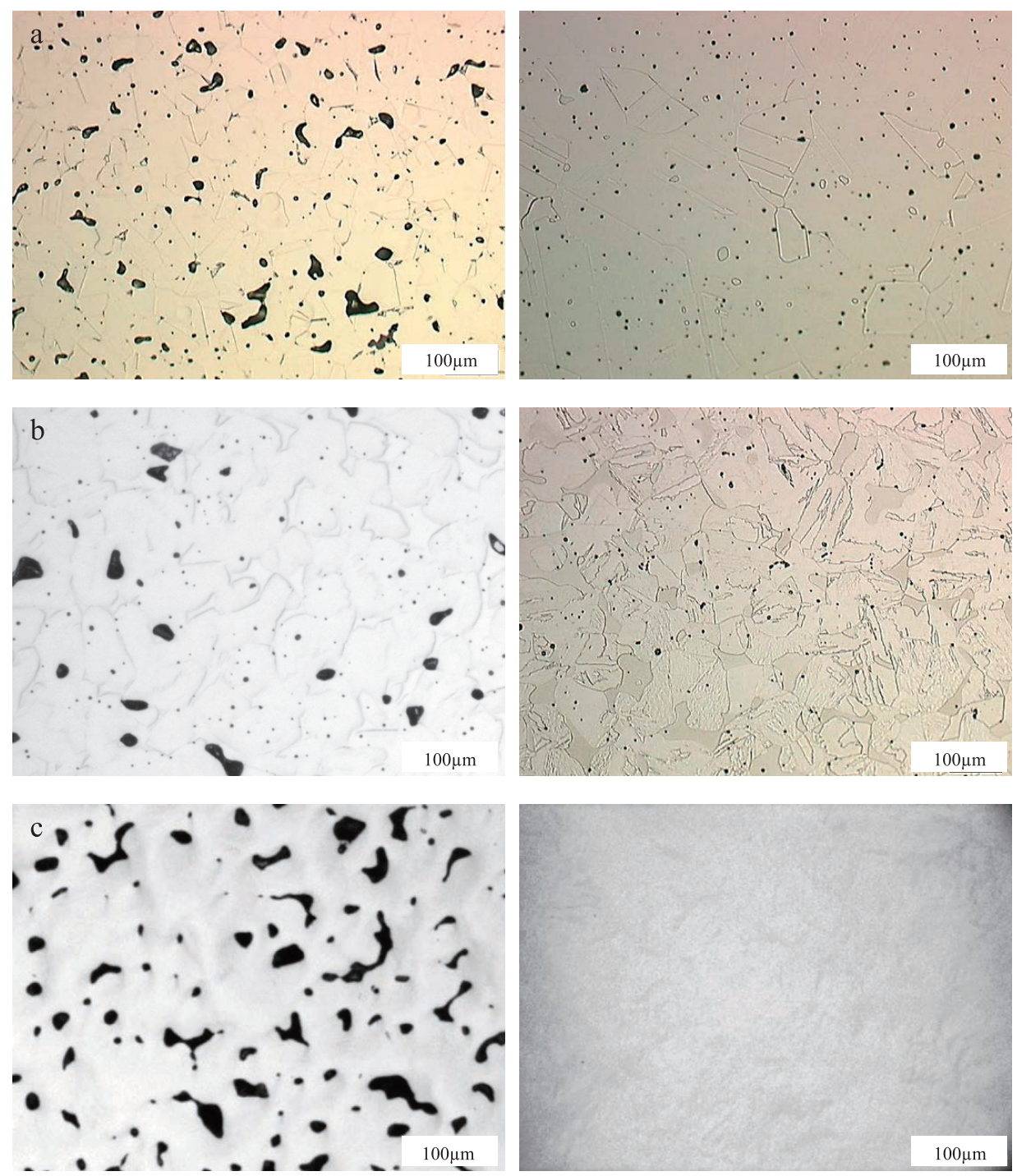

Fig. 6 Sintered and HIPed pore structures for a) 316L, b) 17-4PH, and c) Ti-6Al-4V. 
and for Ti-6-Al-4V there are virtually no porosity visible after HIP. When Ti alloys are processed by MIM it is common to apply HIP to obtain high final density. In this study, hot isostatic pressing was performed at $1150{ }^{\circ} \mathrm{C}$ for $1.5 \mathrm{~h}$ with a pressure of $1000 \mathrm{bar}$.

There is also a second way to obtain high density, and that is by reducing the printed volume of the component. The principle then is to just print the surface layer of the component, and let the bulk of the component consist of dense packed loose powder contained by the printed shell. This technique is referred to as shell-printing. Even though the powder in the bulk is loose, the powder particles are close packed, and not able to move. The principle is illustrated in Fig. 7. A result of that process can be seen in Fig. 8. In this case, a cylinder with height $24 \mathrm{~mm}$ and diameter $24 \mathrm{~mm}$ was produced by only printing a $1 \mathrm{~mm}$ thick surface shell. The image shows pore- and microstructure after sintering, with the surface shell to the left. As can be seen, the addition of organic has an effect on densification. The surface zone has porosity on the same level as for a standard printed specimen, while the bulk has significantly reduced porosity. This is shown in the figure to the right, which displays structure of the bulk. Only small and evenly distributed pores are present, and the densification is almost on the same level as for HIP. Beside the positive effect on density, there are additional benefits with the technique. Since the total printed volume is reduced, both time and amount of organic additive can be reduced. The fact that amount of organic additive can be reduced is important when processing elements sensitive to carbon, e.g. Ti-alloys. This makes debinding of the components easier and faster, both since total amount of $\mathrm{C}$ needed to remove is reduced, but also since all $\mathrm{C}$ is present in the component surface at the start of the process, and does not have to migrate from the bulk.

In Table $2 \mathrm{C}$-content after sintering can be found. The table displays a clear effect of the reduced amount of organic in the shell-printing process, with reduction of final C-level from 0.013 down to 0.003 . Comparing with MPIF 35 standard it can also be seen that both materials are within the limit for $\mathrm{C}$-content.

\section{Mechanical performance}

To investigate mechanical performance of $316 \mathrm{~L}$, tensile test bars were produced, both by standard printing and by shell printing. Geometry was chosen according to ISO2740, and shrinkage during sintering was accounted for in the printing step in order to get the correct final dimensions. The bars were sintered in vacuum with Ar addition at $1380{ }^{\circ} \mathrm{C}$. The results are presented in Table 3. For comparison also minimum and typical values from MPIF 35, metal injection molding standard, are presented. As can be seen that the material is well above minimum levels, and performance match typical MIM values. There is no large difference between standard and shell printed specimens, but shell-printing offers somewhat higher strength and hardness. The MIM standard was chosen as reference since it is most relevant process to use for comparison. Consolidation of the powder is quite similar for inkjet on powder bed and MIM. In both cases, powder particles have not been subjected to any large forces, shrinkage is uniform, and isotropic properties can be expected.

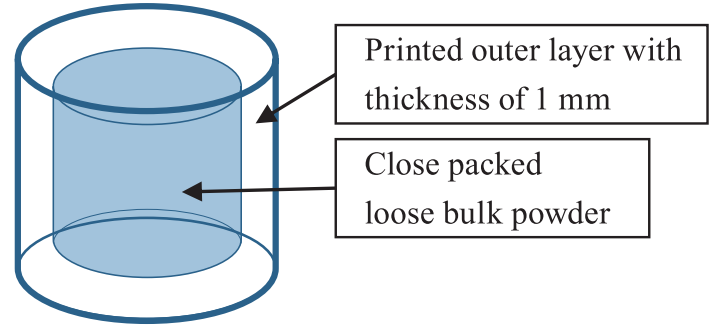

Fig. 7 Principle of shell printing.
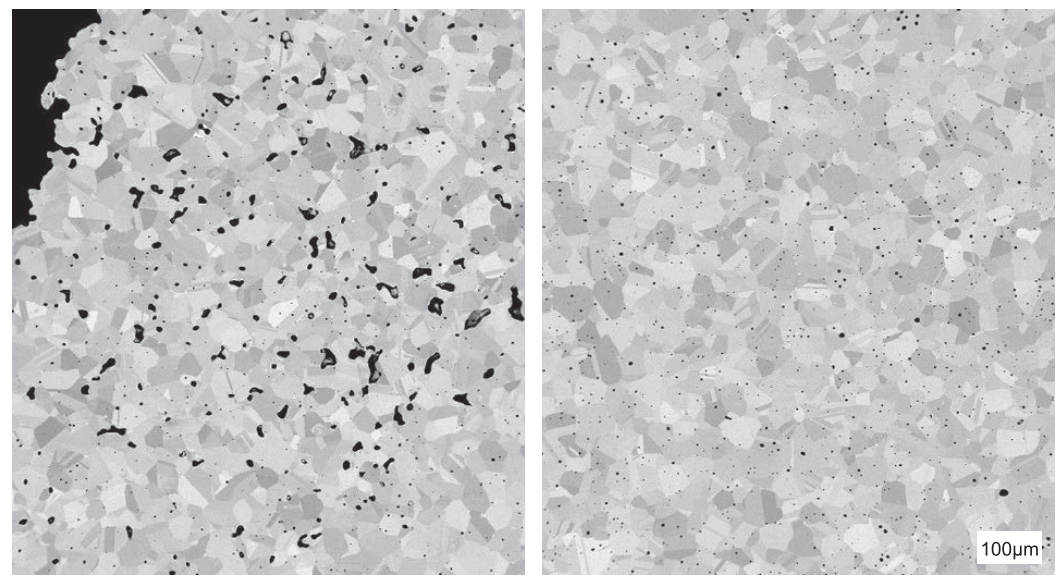

Fig. 8 Pore- and microstructure of bulk after shell-printing and sintering $\left(\mathrm{after}^{2)}\right)$. 
Table 3 Mechanical performance of 316L.

\begin{tabular}{lcccccccc}
\hline & $\begin{array}{c}\text { Density } \\
\mathrm{g} / \mathrm{cm}^{3}\end{array}$ & $\begin{array}{c}\text { Tensile strength } \\
\mathrm{MPa}\end{array}$ & stdv & $\begin{array}{c}\text { Yield strength } \\
\mathrm{MPa}\end{array}$ & stdv & $\begin{array}{c}\text { Elongation } \\
(\%)\end{array}$ & stdv & HRB \\
\hline 316L & 7.67 & 511 & 2.3 & 170 & 1.2 & 58 & 0.74 & 60 \\
Standard print & 7.84 & 528 & 1.7 & 175 & 0.6 & 57 & 1.5 & 62 \\
316L & & & & & & & & \\
Shell print & & & & & & & & \\
MPIF 35, MIM, Minimum & & 520 & & 140 & & 50 & & \\
MPIF 35, MIM, Typical & 7.6 & & & & & &
\end{tabular}

\section{Conclusions}

$316 \mathrm{~L}$ stainless steel can be printed and sintered to $7.7 \mathrm{~g} / \mathrm{cm}^{3}$ density. By utilizing HIP close to full density can be achieved.

Surface roughness after sintering is normally $R_{a} 6$ but can easily be improved with traditional surface treatment processes to $\mathrm{R}_{\mathrm{a}} 3$ or better.

Investigations show that mechanical performance of printed and sintered $316 \mathrm{~L}$ is at the same level as for specimens processed by metal injection molding, and that the material fulfills standard requirements.

$17-4 \mathrm{ph}$ steel can be processed in the same way as $316 \mathrm{~L}$, to close to full density. Mechanical performance can be adjusted with subsequent heat treatments.

Ti-6Al-4V can be printed and sintered with low final impurity levels. By utilizing HIP, full density can be reached.

$\mathrm{Cu}$ and $\mathrm{Ag}$ are possible to utilize in the Digital Metal process

By utilizing shell-printing technique density can be improved. For $316 \mathrm{~L}$ a density level of $7.84 \mathrm{~g} / \mathrm{cm}^{3}$ can be achieved without post-sintering processing.

The higher density obtained by shell-printed somewhat increases strength and hardness.

Shell-printing leads to reduction of total C-content after sintering.

\section{References}

1) R. Frykholm, B.-G. Andersson, R. Carlström: "Progress of Precision Ink jet printing on powder bed", Proceedings Euro PM2015, Reims, France, 4-7 Oct, 2015.

2) P. V. Muterlle, M. Perina, A. Molinari: "Mechanical properties and corrosion resistance of vacuum sintered MIM 316L stainless steel containing delta ferrite", Powder Injection Moulding International, 4(2) (2010) 66-70.

3) R. M. German, J. L. Johnson: "Metal powder injection molding of copper and copper alloys for microelectronics heat dissipation”, Int. J. of Powder Metallurgy, 43(5) (2007) 55-63. 